\title{
13.
}

\section{ON THE THEORY OF LINEAR TRANSFORMATIONS.}

[From the Cambridge Mathematical Journal, vol. Iv. (1845), pp. 193-209.]

THE following investigations were suggested to me by a very elegant paper on the same subject, published in the Journal by $\mathrm{Mr}$ Boole. The following remarkable theorem is there arrived at. If a rational homogeneous function $U$, of the $n^{\text {th }}$ order with the $m$ variables $x, y \ldots$, be transformed by linear substitutions into a function $V$ of the new variables, $\xi, \eta \ldots$; if, moreover, $\theta U$ expresses the function of the coefficients of $U$, which, equated to zero, is the result of the elimination of the variables from the series of equations $d_{x} U=0, d_{y} U=0$, \&c., and of course $\theta V$ the analogous function of the coefficients of $V$ : then $\theta V=E^{n a} \cdot \theta U$, where $E$ is the determinant formed by the coefficients of the equations which connect $x, y \ldots$ with $\xi, \eta \ldots,{ }^{1}$ and $\alpha=(n-1)^{m-1}$. In attempting to demonstrate this very beautiful property, it occurred to me that it might be generalised by considering for the function $U$, not a homogeneous function of the $n^{\text {th }}$ order between $m$ variables, but one of the same order, containing $n$ sets of $m$ variables, and the variables of each set entering linearly. The form which $\mathrm{Mr}$ Boole's theorem thus assumes is $\theta V=E_{1}{ }^{a} \cdot E_{2}{ }^{a} \ldots E_{p}{ }^{a} \cdot \theta U$. This it was easy to demonstrate would be true, if $\theta U$ satisfied a certain system of partial differential equations. I imagined at first that these would determine the function $\theta U$, (supposed, in analogy with $\mathrm{Mr}$ Boole's function, to represent the result of the elimination of the variables from $d_{x_{1}} U=0, d_{y_{1}} U=0, \ldots d_{x_{2}} U=0, \&$ c.) : this I afterwards found was not the case; and thus I was led to a class of functions, including a particular case the function $\theta U$, all of them possessed of the same characteristie property. The system of partial differential equations was without difficulty replaced by a more fundamental system of equations, upon which, assumed as definitions, the theory appears to me naturally to depend; and it is this view of it which I intend partially to develope in the present paper.

1 The value of a was left undetermined, but Mr Boole has since informed me, he was acquainted wit it at the time his paper was written; and has given it in a subsequent paper. 
I have already employed the notation

$$
\mid \begin{array}{llll}
\alpha, & \beta, & \gamma, & \delta, \ldots \\
\alpha^{\prime}, & \beta^{\prime}, & \gamma^{\prime}, & \delta^{\prime} \\
\alpha^{\prime \prime}, & \beta^{\prime \prime}, & \gamma^{\prime \prime}, & \delta^{\prime \prime} \\
\vdots &
\end{array}
$$

(where the number of horizontal rows is less than that of vertical ones) to denote the series of determinants,

$$
\left|\begin{array}{lll}
\alpha, & \beta, & \gamma, \ldots \\
\alpha^{\prime}, & \beta^{\prime}, & \gamma^{\prime} \\
\alpha^{\prime \prime}, & \beta^{\prime \prime}, & \gamma^{\prime \prime} \\
\vdots & &
\end{array}\right|
$$

which can be formed out of the above quantities by selecting any system of vertical rows; these different determinants not being connected together by the sign + , or in any other manner, but being looked upon as perfectly separate.

The fundamental theorem for the multiplication of determinants gives, applied to these, the formula

$$
\begin{array}{llll}
A, & B, & C, & D, \ldots \\
A^{\prime}, & B^{\prime}, & C^{\prime}, & D^{\prime}, \\
A^{\prime \prime}, & B^{\prime \prime}, & C^{\prime \prime}, & D^{\prime \prime}, \\
\vdots &
\end{array}\|=E\| \begin{array}{llll}
\alpha, & \beta, & \gamma, & \delta, \cdots \\
\alpha^{\prime}, & \beta^{\prime}, & \gamma^{\prime}, & \delta^{\prime}, \\
\alpha^{\prime \prime}, & \beta^{\prime \prime}, & \gamma^{\prime \prime}, & \delta^{\prime \prime}, \\
\vdots & &
\end{array}
$$

where

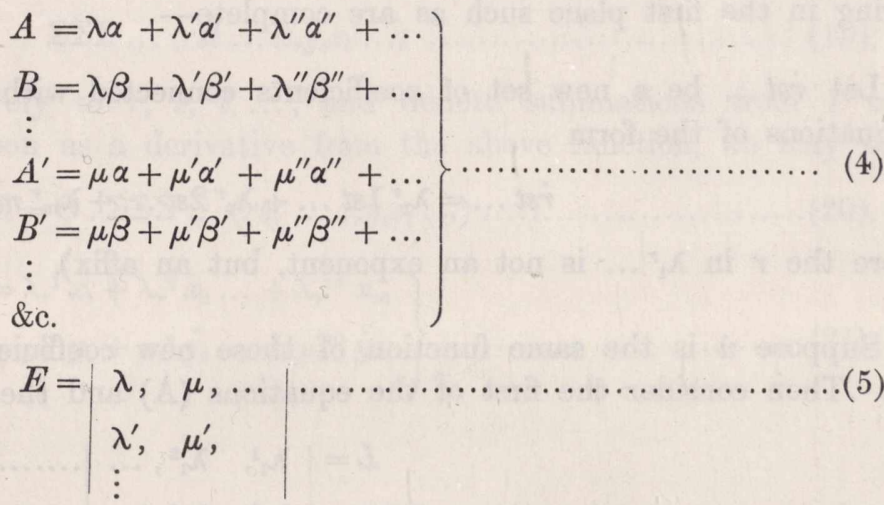

and the meaning of the equation is, that the terms on the first side are equal, each to each, to the terms on the second side.

This preliminary theorem being explained, consider a set of arbitrary coefficients, represented by the general formula

$$
r s t \ldots
$$

in which the number of symbolical letters $r, s, \ldots$ is $n$, and where each of these is supposed to assume all integer values, from 1 to $m$ inclusively.

c. 
Let

$$
\alpha s_{,}^{\prime} t_{1}^{\prime} \ldots, \alpha s_{1}^{\prime \prime} t_{1}^{\prime \prime} \ldots,
$$

represent the whole series, taken in any order, in which the first symbolical letter is $\alpha$. Similarly,

$$
r_{11}{ }^{\prime} \alpha t_{1}{ }^{\prime} \ldots, \quad r_{1 /}{ }^{\prime \prime} \alpha t_{\prime \prime}{ }^{\prime \prime}, \ldots
$$

the whole series of those in which the second symbolical letter is $\alpha$, and so on.

Imagine a function $u$ of the coefficients, which is simultaneously of the forms

$$
\begin{aligned}
& u=H_{p}\left\|\begin{array}{cc}
1 s_{\prime}^{\prime} t_{1}^{\prime} \ldots, & 1 s_{,}^{\prime \prime} t_{\prime}^{\prime \prime} \ldots, \ldots \\
2 s_{,}^{\prime} t_{1}^{\prime} \ldots, & 2 s_{,}^{\prime \prime} t_{,}^{\prime \prime} \ldots, \\
\vdots &
\end{array}\right\|
\end{aligned}
$$

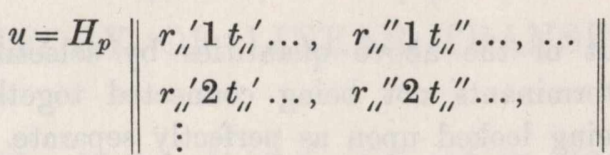

\&c.; in which $H_{p}$ denotes a rational homogeneous function of the order $p$. The function $H$ is not necessarily supposed to be the same in the above equations, and in point of fact it will not in general be so. The number of equations is of course $=n$.

The function $u$, whose properties we proceed to investigate, may conveniently be named a "Hyperdeterminant." Any function satisfying any of the equations (A), without satisfying all of them, will be an "Incomplete Hyperdeterminant." But, considering in the first place such as are complete-

Let $\dot{r} s t \ldots$ be a new set of coefficients connected with the former ones by a system of equations of the form

$$
\dot{r} s t \ldots=\lambda_{1}^{r} 1 s t \ldots+\lambda_{2}^{r} 2 s t \ldots+\lambda_{m}{ }^{r} m s t \ldots
$$

(where the $r$ in $\lambda_{1}^{r} \ldots$ is not an exponent, but an affix).

Suppose $\dot{u}$ is the same function of these new coefficients that $u$ was of the former ones. Then consider the first of the equations (A) and the equation (3), and writing

$$
L=\mid \begin{array}{ll}
\lambda_{1}^{1}, & \lambda_{1}{ }^{2}, \ldots \\
\lambda_{2}{ }^{1}, & \lambda_{2}{ }^{2}, \\
\vdots &
\end{array}
$$

we have immediately the equation

$$
\dot{u}=L^{p} \cdot u
$$

Consider the new set of coefficients

$$
\ddot{r} \dot{s} t \ldots=\mu_{1}^{8} \dot{r} 1 t \ldots+\mu_{2}^{8} \dot{r} 2 t \ldots+\ldots+\mu_{m}^{8} \dot{r} m t \ldots
$$


and $\ddot{u}$ the analogous function of these; then, from the second of the equations (A) and the equation (3), and writing

$$
\begin{gathered}
M=\left|\begin{array}{cc}
\mu_{1}^{1}, & \mu_{1}^{2}, \ldots \\
\mu_{2}{ }^{1}, & \mu_{2}{ }^{2}, \\
\vdots &
\end{array}\right| \\
\ddot{u}=M^{p} \cdot \ddot{u}=L^{p} M^{p} \cdot u
\end{gathered}
$$

In like manner, considering the new coefficients $\ddot{r} \ddot{s} \dot{t} \ldots$, where

$$
\ddot{r} \ddot{s} \dot{t} \ldots=\nu_{1}^{t} \ddot{r} \dot{s} 1 \ldots+\nu_{2}^{t} \ddot{r} \dot{s} 2 \ldots \ldots+\nu_{n}^{t} \ddot{r} \ddot{s} m \ldots
$$

the new function $\dddot{u}$, and the quantity $N$ given by

$$
N=\left|\begin{array}{ll}
\nu_{1}^{1}, & \nu_{1}^{2}, \ldots \\
\nu_{2}^{1}, & \nu_{2}^{2}, \\
\vdots &
\end{array}\right|
$$

we have, as before,

$$
\begin{aligned}
\dddot{u} & =N^{p} \ddot{u}=L^{p} M^{p} N^{p} u \\
\dddot{u} & =L^{p} M^{p} N^{p} u \ldots \ldots \ldots \ldots \ldots
\end{aligned}
$$

whence, generally, denoting the last result by $u^{\prime}$,

$$
u^{\prime}=L^{p} M^{p} N^{p} \ldots u
$$

Consider now the function

$$
\Sigma \Sigma \Sigma \ldots\left(r s t \ldots x_{r} y_{s} z_{t} \ldots\right)
$$

where the $\Sigma$ 's refer successively to $r, s, t, \ldots$, and denote summations from 1 to $m$ inclusively. If $u$ be looked upon as a derivative from the above function, we may write

$$
u=\Theta . \Sigma \Sigma \Sigma \ldots\left(r s t \ldots x_{r} y_{s} z_{t} \ldots\right)
$$

Assume

$$
\left.\begin{array}{l}
x_{r}=\lambda_{r}{ }^{1} \dot{x}_{1}+\lambda_{r}{ }^{2} \dot{x}_{2} \ldots+\lambda_{r}{ }^{m} \dot{x}_{m} \\
y_{s}=\mu_{s}{ }^{1} y_{1}+\mu_{s}{ }^{2} \dot{y}_{2} \ldots+\mu_{s}{ }^{m} \dot{y}_{m}
\end{array}\right\}
$$

It is easy to obtain

$\Sigma \Sigma \Sigma \ldots\left(r s t \ldots x_{r} y_{s} z_{t} \ldots\right)=\Sigma \Sigma \Sigma \ldots\left(\dot{r} s t \ldots \dot{x}_{r} y_{s} z_{t} \ldots\right), \ldots=\Sigma \Sigma \Sigma \ldots\left(\dot{r} \ddot{s} \dot{t} \ldots \dot{x}_{r} \dot{y}_{s} \dot{z}_{t} \ldots\right) \ldots \ldots(22)$ and the formula for $(u)$ becomes

$$
\Theta \Sigma \Sigma \Sigma \ldots\left(\ddot{r} \ddot{s} \dot{t} \ldots \dot{x}_{r} \dot{y}_{s} \dot{z}_{t}\right) \ldots=L^{p} M^{p} N^{p} \ldots \Theta \Sigma \Sigma \Sigma \ldots\left(r s t \ldots x_{r} y_{s} z_{t}\right)
$$

Proceeding to obtain the expression of the coefficients $\dot{r} \dot{s} t \ldots$ in terms of the coefficients rst..., we have

$$
i \ddot{s} \dot{s} \ldots=\Sigma \Sigma \Sigma \ldots\left(\lambda_{f}{ }^{r} \mu_{l j}{ }^{s} \nu_{h}{ }^{t} \ldots f g h \ldots\right)
$$


where the $\Sigma$ 's refer successively to $f, g, h, \ldots$, denoting summations from 1 to $m$ inclusively. Having this equation, it is perhaps as well to retain

$$
u^{\prime}=L^{p} M^{p} N^{p} \ldots u
$$

instead of $(B, 2)$, that form being principally useful in showing the relation of the function $u$ to the theory of the transformation of functions.

It may immediately be seen, that in the equations (B), (C) we may, if we please, omit any number of the marks of variation ('), omitting at the same time the corresponding signs $\Sigma$, and the corresponding factors of the series $L, M, N \ldots$

Also, if $u$ be such as only to satisfy some of the equations (A); then, if in the same formulæ we omit the corresponding marks ('), summatory signs, and terms of the series $L, M, N \ldots$, the resulting equations are still true.

From the formulæ (A) we may obtain the partial differential equations

$$
\begin{aligned}
& \Sigma \Sigma \ldots\left(\text { ast } \ldots \frac{d}{d \beta s t \ldots}\right) u=0, \text { or } p u, \\
& \Sigma \Sigma \ldots\left(\text { rat } \ldots \frac{d}{d r \beta t \ldots}\right) u=0, \text { or } p u,
\end{aligned}
$$

according as $\alpha$ is not equal, or is equal, to $\beta$;

and so on: the summatory signs referring in every case to those of the series $r, s, t, \ldots$ which are left variable, and extending from 1 to $m$ inclusively.

To demonstrate this, consider the general form of $u$, as given by the first of the equations (A). This is evidently composed of a series of terms, each of the form

in which

$$
\begin{aligned}
& c P Q R \ldots(p \text { factors }),
\end{aligned}
$$

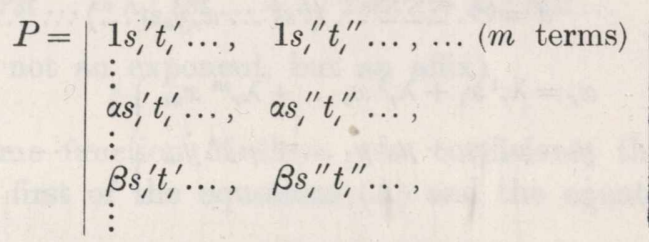

$Q, R$, \&c. being of the same form; and we have

and

$$
\begin{aligned}
& \Sigma \Sigma \ldots\left(\text { ast } \ldots \frac{d}{d \beta s t \ldots}\right) u=c Q R \ldots \Sigma \Sigma \ldots\left(\text { ast } \ldots \frac{d}{d \beta s t \ldots}\right) P+\& c .+\& c .
\end{aligned}
$$

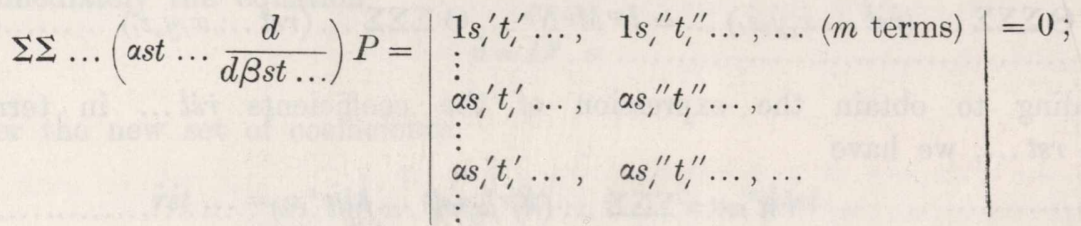


so that all the terms on the second side of the equation vanish. If, however, $\beta=\alpha$,

$$
\Sigma \Sigma \ldots\left(\alpha s t \ldots \frac{d}{d \beta s t \ldots}\right) P=P
$$

whence, on the second side, we have

$$
\begin{aligned}
c Q R \ldots P+c P R \ldots Q+\& c . & =p \cdot c P Q R \ldots+\& c .+\& c . \\
& =p U
\end{aligned}
$$

or the theorem in question is proved.

In the case of an incomplete hyperdeterminant, the corresponding systems of equations are of course to be omitted. In every case it is from these equations that the form of the function $u$ is to be investigated; they entirely replace the system (A).

A very important case of the general theory is, when we suppose the coefficients $r s t . .$. to have the property $r^{\prime} s^{\prime} t^{\prime} \ldots=r^{\prime \prime} s^{\prime \prime} t^{\prime \prime} \ldots$, whenever $r^{\prime} s^{\prime} t^{\prime} \ldots$ and $r^{\prime \prime} s^{\prime \prime} t^{\prime \prime} \ldots$ denote the same combination of letters; and also that the coefficients $\lambda$ are equal to the coefficients $\mu, \nu \ldots$, each to each. In this case the coefficients $\ddot{r} \dot{s} t \ldots$ have likewise the same property, viz. that $\dot{r}^{\prime \prime} \dot{s}^{\prime \prime} \dot{t}^{\prime \prime} \ldots=\dot{r}^{\prime} \dot{s}^{\prime} \dot{t}^{\prime} \ldots$, whenever $r^{\prime} s^{\prime} t^{\prime} \ldots$ and $r^{\prime \prime} s^{\prime \prime} t^{\prime \prime} \ldots$ denote the same combination of letters.

The equations $(B, 1),(B, 2)$, become in this case

$$
u^{\prime}=L^{n p} \cdot u
$$

$$
\Theta \Sigma \Sigma \Sigma \ldots\left\{\frac{[n]^{n}}{[\alpha]^{\alpha}[\beta]^{\beta} \ldots} \dot{r} \dot{s} \dot{t} \ldots \dot{x}_{r} \dot{x}_{g} \dot{x}_{t} \ldots\right\}=L^{n p} . \Theta\left\{\frac{[n]^{n}}{[\alpha]^{\alpha}[\beta]^{\beta} \ldots} r s t \ldots x_{r} x_{s} x_{t} \ldots\right\} \ldots . .(\mathrm{B}, 4) \text {, }
$$

where only different combinations of values are to be taken for $r, s, t, \ldots$ and $\alpha, \beta, \ldots$ express how often the same number occurs in the series. In the equation (C), $\mu, \nu$ must be replaced by $\lambda$, the equations (D) are no longer satisfied; the equations (A) reduce themselves to a single one, (so that there can be no question here of incomplete hyperdeterminants): but this is no longer sufficient to determine the function sought after. For this reason, the particular case, treated separately, would be far more difficult than the general one; but the formulæ of the general case being first established, these apply immediately to the particular one ${ }^{1}$. The case in question may be defined as that of symmetrical hyperdeterminants, (a denomination already adopted for ordinary determinants). It would be easily seen what on the same principle would be meant by partially symmetrical hyperdeterminants.

I have not yet succeeded in obtaining the general expression of a hyperdeterminant; the only cases in which I can do so are the following: I. $p=1, n$ even, (if $n$ be odd, there only exist incomplete hyperdeterminants). II. $p=2, m=2, n$ even. III. $p=3$, $m=2, n=4$.

I. The first case is, in fact, that of the functions considered at the termination of a paper in the Cambridge Philosophical Transactions, vol. vIII. part I. [12]; though at that time I was quite unacquainted with the general theory.

\footnotetext{
1 See concluding paragraph of this paper.
} 
Using the notation there employed, we have

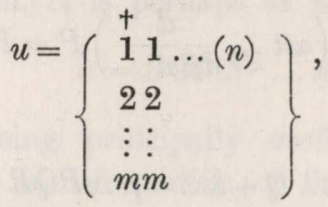

a complete hyperdeterminant when $n$ is even; and when $n$ is odd the functions

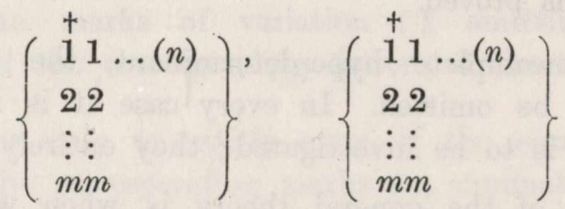

are each of them incomplete hyperdeterminants.

(A) In the case of $n=2$, the complete hyperdeterminant is simply the ordinary determinant

$$
\left|\begin{array}{lll}
11, & 12, \ldots 1 m \\
21, & 22, \ldots 2 m \\
\vdots & \\
m 1, & m 2, \ldots m m
\end{array}\right|
$$

Stating the general conclusion as applied to this case, which is a very well known one,

"If the function

$$
U=\Sigma \Sigma\left(r s . x_{r} y_{s}\right)
$$

be transformed into a similar function

$$
\sum \Sigma\left(\dot{r} \dot{s}, \dot{x}_{r} \dot{y}_{s}\right),
$$

by means of the substitutions

$$
\begin{aligned}
& x_{r}=\lambda_{r}{ }^{1} \dot{x}_{1}+\lambda_{r}{ }^{2} \dot{x}_{2} \ldots+\lambda_{r}{ }^{m} \dot{x}_{m}, \\
& y_{s}=\mu_{s}{ }^{1} \dot{y}_{1}+\mu_{s}{ }^{2} \dot{y}_{2} \ldots+\mu_{s}{ }^{m} \dot{y}_{m} ;
\end{aligned}
$$

then

$$
\left|\begin{array}{cc}
i i, & i \dot{2}, \ldots \\
\dot{2} \dot{1}, & \dot{2} \dot{2}, \\
\vdots &
\end{array}\right|=\left|\begin{array}{cc}
\lambda_{1}{ }^{1}, & \lambda_{2}{ }^{2}, \ldots \\
\lambda_{1}{ }^{2}, & \lambda_{2}{ }^{2}, \\
\vdots &
\end{array}\right|\left|\begin{array}{cc}
\mu_{1}^{1}, & \mu_{2}{ }^{1}, \ldots \\
\mu_{1}{ }^{2}, & \mu_{2}{ }^{2}, \\
\vdots &
\end{array}\right|\left|\begin{array}{cc}
11, & 12, \ldots \\
21, & 22, \\
\vdots &
\end{array}\right|
$$

Also, by what has preceded,

$$
\ddot{r} \dot{s}=\Sigma \Sigma\left(\lambda_{f}{ }^{r} \cdot \mu_{g}{ }^{s} \cdot f g\right) ;
$$

so that the theorem is easily seen to amount to the following one- "If the terms of a determinant of the $m^{\text {th }}$ order be of the form $\Sigma_{r} \Sigma_{s}\left(r s . x_{r \rho} y_{s \sigma}\right), r, s$ extending as before, from 1 to $m$ inclusively, the determinant itself is the product of three determinants; the first formed with the coefficients $r s$, the second with the quantities $x$, and the third with the quantities $y . "$ 
In a following number of the Journal I shall prove, and apply to the theories of Maxima and Minima and of Spherical Coordinates, (I may just mention having obtained, in an elegant form, the formulæ for transforming from one oblique set of coordinates to another oblique one) the more general theorem,

"If $k$ be the order of the determinant formed as above, the determinant itself is a quadratic function, its coefficients being determinants formed with the coefficients $r s$, its variables being determinants formed respectively with the variables $x$ and the variables $y$; and the number of variables in each set being the number of combinations of $k$ things out of $m$, (=1 if $k=m$; if $k>m$ the determinant vanishes)."

I shall give in the same paper the demonstration of a very beautiful theorem, rather relating, however, to determinants than to quadratic functions, proved by Hesse in a Memoir in Crelle's Journal, vol. xx., "De curvis et superficiebus secundi ordinis;" and from which he has deduced the most interesting geometrical results. Another Memoir, by the same author, Crelle, vol. xxviII., "Ueber die Elimination der Variabeln aus drei algebraischen Gleichungen vom zweiter Grade mit zwei Variabeln," though relating in point of fact rather to functions of the third order, contains some most important results. A few theorems on quadratic functions, belonging, however, to a different part of the subject, will be found in my paper already quoted in the Cambridge Philosophical Transactions [12]; and likewise in a paper in the Journal, Chapters in the Algebraical Geometry of $n$ dimensions [11].

I shall, just before concluding this case, write down the particular formula corresponding to three variables, and for the symmetrical case. It is, as is well known, the theorem,

"If

$$
U=A x^{2}+B y^{2}+C z^{2}+2 F y z+2 G x z+2 H x y
$$

be transformed into

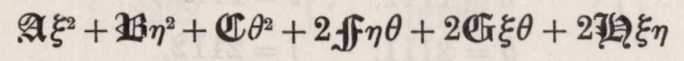

by means of

$$
\begin{aligned}
& x=\alpha \xi+\beta \eta+\gamma \theta, \\
& y=\alpha^{\prime} \xi+\beta^{\prime} \eta+\gamma^{\prime} \theta, \\
& z=\alpha^{\prime \prime} \xi+\beta^{\prime \prime} \eta+\gamma^{\prime \prime} \theta,
\end{aligned}
$$

then

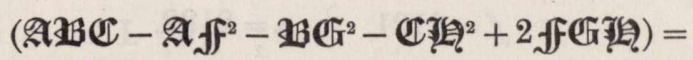

$\left(\alpha \beta^{\prime} \gamma^{\prime \prime}-\alpha \beta^{\prime \prime} \gamma^{\prime}+\alpha^{\prime} \beta^{\prime \prime} \gamma-\alpha^{\prime} \beta \gamma^{\prime \prime}+\alpha^{\prime \prime} \beta \gamma^{\prime}-\alpha^{\prime \prime} \beta^{\prime} \gamma\right)^{2}\left(A B C-A F^{2}-B G^{2}-C H^{2}+2 F G H\right) . "$

(B) Let $n=3$, and for greater simplicity $m=2$; write

$$
\begin{array}{ll}
a=111, & e=112, \\
b=211, & f=212, \\
c=121, & g=122, \\
d=221, & h=222,
\end{array}
$$

so that $U=a x_{1} y_{1} z_{1}+b x_{2} y_{1} z_{1}+c x_{1} y_{2} z_{1}+d x_{2} y_{2} z_{1}+e x_{1} y_{1} z_{2}+f x_{2} y_{1} z_{2}+g x_{1} y_{2} z_{2}+h x_{2} y_{2} z_{2}$. 
There is no complete hyperdeterminant (i.e. for $p=1$ ), and the incomplete ones are

$$
\begin{aligned}
& a h-b g-c f+d e=u_{,}, \text {suppose, } \\
& a h-d e-b g+c f=u_{1 /}, \\
& a h-c f-d e+b g=u_{\mu \prime} .
\end{aligned}
$$

Thus, suppose the transforming equations are

$$
\begin{aligned}
& x_{1}=\lambda_{1}{ }^{1} \dot{x}_{1}+\lambda_{1}{ }^{2} \dot{x}_{2}, \\
& x_{2}=\lambda_{2}{ }^{1} \dot{x}_{1}+\lambda_{2}{ }^{2} \dot{x}_{2} ; \\
& y_{1}=\mu_{1}{ }^{1} \dot{y}_{1}+\mu_{1}{ }^{2} \dot{y}_{2}, \\
& y_{2}=\mu_{2}{ }^{1} \dot{y}^{1}+\mu_{2}{ }^{2} \dot{y}_{2} ; \\
& z_{1}=\nu_{1}{ }^{1} \dot{z}_{1}+\nu_{1}{ }^{2} \dot{z}_{2}, \\
& z_{2}=\nu_{2}{ }^{1} \dot{z}_{1}+\nu_{2}{ }^{2} \dot{z}_{2} ;
\end{aligned}
$$

then

$$
\begin{aligned}
& \dddot{u}_{1}=\left(\mu_{1}^{1} \mu_{2}{ }^{2}-\mu_{2}{ }^{1} \mu_{1}{ }^{2}\right)\left(\nu_{1}^{1} \nu_{2}{ }^{2}-\nu_{2}{ }^{1} \nu_{1}{ }^{2}\right) u_{1} \text {, where } y, z \text { are changed } \\
& \dddot{u}_{\text {" }}=\left(\nu_{1}{ }^{1} \nu_{2}{ }^{2}-\nu_{2}{ }^{1} \nu_{1}{ }^{2}\right)\left(\lambda_{1}{ }^{1} \lambda_{2}{ }^{2}-\lambda_{2}{ }^{1} \lambda_{1}{ }^{2}\right) u_{1 \prime}, \quad, \quad z, x \\
& \dddot{u}_{\text {"I }}=\left(\lambda_{1}{ }^{1} \lambda_{2}{ }^{2}-\lambda_{2}{ }^{1} \lambda_{1}{ }^{2}\right)\left(\mu_{1}{ }^{1} \mu_{2}{ }^{2}-\mu_{2}{ }^{1} \mu_{1}{ }^{2}\right) u_{\mu \prime}, \quad, \quad x, y
\end{aligned}
$$

We might also have assumed

$$
\begin{aligned}
& u_{\iota}=a d-b c, \text { or } e h-g f, \\
& u_{\prime \prime}=a f-b e, \text { or } c h-d g, \\
& u_{u}=a g-c e, \text { or } b h-d f,
\end{aligned}
$$

but these are ordinary determinants.

$$
\begin{array}{rll}
(C) n=4, m=2 . & a=1111, & i=1112, \\
b=2111, & j=2112, \\
c=1211, & k=1212, \\
d=2211, & \quad l=2212, \\
e=1121, & m=1122, \\
f=2121, & n=2122, \\
g=2211, & o=2212, \\
h=2221, & p=2222 . \\
U= & a x_{1} y_{1} z_{1} w_{1}+b x_{2} y_{2} z_{1} w_{1}+c x_{1} y_{2} z_{1} w_{1}+d x_{2} y_{2} z_{1} w_{1} \\
+e x_{1} y_{1} z_{2} w_{1}+f x_{2} y_{1} z_{2} w_{1}+g x_{2} y_{2} z_{1} w_{1}+h x_{2} y_{2} z_{2} w_{1} \\
+i x_{1} y_{1} z_{1} w_{2}+j x_{2} y_{1} z_{1} w_{2}+k x_{1} y_{2} z_{1} w_{2}+l x_{2} y_{2} z_{1} w_{2} \\
+m x_{1} y_{1} z_{2} w_{2}+n x_{2} y_{1} z_{2} w_{2}+o x_{2} y_{2} z_{1} w_{2}+p x_{2} y_{2} z_{2} w_{2},
\end{array}
$$

we have

$$
u=a p-b o-c n+d m-e l+f k+g j-h i
$$


so that, with the same sets of transforming equations as above, and the additional one,

$$
\begin{aligned}
& w_{1}=\rho_{1}^{1} \dot{w}_{1}+\rho_{1}{ }^{2} \dot{w}_{2}, \\
& w_{n}=\rho_{2}{ }^{1} \dot{w}_{1}+\rho_{2}{ }^{2} \dot{w}_{2},
\end{aligned}
$$

we have

$$
\dddot{u}=\left(\lambda_{1}{ }^{1} \lambda_{2}{ }^{2}-\lambda_{2}{ }^{1} \lambda_{1}{ }^{2}\right)\left(\mu_{1}^{1} \mu_{2}{ }^{2}-\mu_{2}{ }^{1} \mu_{1}{ }^{2}\right)\left(\nu_{1}{ }^{1} \nu_{2}{ }^{2}-\nu_{2}{ }^{1} \nu_{1}{ }^{2}\right)\left(\rho_{1}{ }^{1} \rho_{2}{ }^{2}-\rho_{2}{ }^{1} \rho_{1}{ }^{2}\right) \cdot u ;
$$

this is important when viewed in reference to a result which will presently be obtained.

If we take the symmetrical case, we have

$$
U=\alpha x^{4}+4 \beta x^{3} y+6 \gamma x^{2} y^{2}+4 \delta x y^{3}+\epsilon y^{4}
$$

which is transformed into

$$
U^{\prime}=\alpha^{\prime} x^{\prime 4}+4 \beta^{\prime} x^{\prime 3} y^{\prime}+6 \gamma^{\prime} x^{\prime 2} y^{\prime 2}+4 \delta^{\prime} x^{\prime} y^{\prime 3}+\epsilon^{\prime} y^{\prime 4},
$$

by means of

$$
\begin{aligned}
& x=\lambda x^{\prime}+\mu y^{\prime}, \\
& y=\lambda, x^{\prime}+\mu, y^{\prime} ;
\end{aligned}
$$

then, if

$$
\begin{array}{ll}
\text { then, if } & u=\alpha \epsilon-4 \beta \delta+3 \gamma^{2}, \\
& u^{\prime}=\alpha^{\prime} \epsilon^{\prime}-4 \beta^{\prime} \delta^{\prime}+3 \gamma^{\prime 2}, \\
\text { we have } & u^{\prime}=(\lambda \mu,-\lambda, \mu)^{4} \cdot u .
\end{array}
$$

II. Where $p=2, m=2, n$ is odd.

The expression

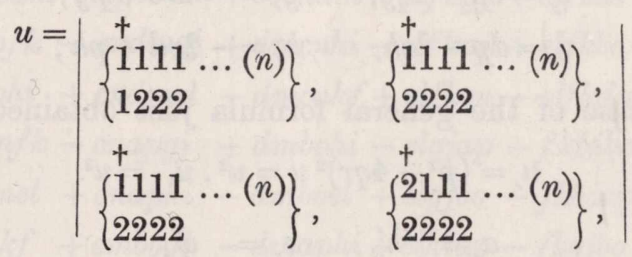

is a complete hyperdeterminant; and that over whichever row the mark $(\dagger)$ of nonpermutation is placed. The different expressions so obtained are not, however, all of them independent functions: thus, in the following example, where $n=3$, the three functions are absolutely identical.

(A). $n=3$, notation as in I. $(B)$. $u=a^{2} h^{2}+b^{2} g^{2}+c^{2} f^{2}+d^{2} e^{2}-2 a h b g-2 a h c f-2 a h d e-2 b g c f-2 b g d e-2 c f d e+4 a d f g+4 b e c h$, and then $\quad \because \ddot{u}=\left(\lambda_{1}{ }^{1} \lambda_{2}{ }^{2}-\lambda_{2}{ }^{1} \lambda_{1}{ }^{2}\right)^{2}\left(\mu_{1}{ }^{1} \mu_{2}{ }^{2}-\mu_{2}{ }^{1} \mu_{1}{ }^{2}\right)^{2}\left(\nu_{1}{ }^{1} \nu_{2}{ }^{2}-\nu_{2}{ }^{1} \nu_{1}^{2}\right)^{2} u$.

This is in many respects an interesting example. We see that the function $u$ may be expressed in the three following forms:

$$
\begin{aligned}
& u=(a h-b g-c f+d e)^{2}+4(a d-b c)(f g-e h) . \\
& u=(a h-b g-d e+c f)^{2}+4(a f-b e)(d g-c h) . \\
& u=(a h-c f-d e+b g)^{2}+4(a g-c e)(d f-b h) .
\end{aligned}
$$

C. 
which are indeed the direct results of the general form above given, the sign ( $\dagger$ ) being placed in succession over the different columns: and the three forms, as just remarked, are in this case identical.

We see from the first of these that $u$ is of the second or third, from the second that $u$ is of the first or third, from the third that $u$ is of the first or second of the three following forms:

$$
u=H_{2}\left\|\begin{array}{l}
a, b, c, d \\
e, f, g, h
\end{array}\right\|, \quad u=H_{2}\left\|\begin{array}{l}
a, b, e, f \\
c, d, g, h
\end{array}\right\|, \quad u=H_{2}\left\|\begin{array}{l}
a, c, e, g \\
b, d, f, h
\end{array}\right\|,
$$

which is as it should be.

The following is a singular property of $u$.

Let

$$
a^{\prime}=\frac{1}{2} \frac{d u}{d a}, \quad b^{\prime}=\frac{1}{2} \frac{d u}{d b}, \ldots \ldots h^{\prime}=\frac{1}{2} \frac{d u}{d h},
$$

then, $u^{\prime}$ being the same function of these new coefficients that $u$ is of the former ones,

$$
u^{\prime}=u^{3} \text {. }
$$

To prove this, write

$$
\begin{array}{cc}
p=a h-b g-c f+d e, & q=(a d-b c), \quad r=e h-f g ; \\
a_{1}=a p-2 q e, & e_{1}=-2 r a+p e, \\
b_{\imath}=b p-2 q f, & f_{l}=-2 r b+p f, \\
c_{1}=c p-2 q g, & g_{1}=-2 r c+p g, \\
d_{l}=d p-2 q h, & h_{l}=-2 r d+p h ;
\end{array}
$$

we have, as a particular case of the general formula just obtained,

$$
u_{l}=\left(p^{2}-4 q r\right)^{2} u=u^{2} \cdot u,=u^{3} .
$$

Also

$$
\begin{array}{ll}
a_{1}=h^{\prime}, & e_{1}=d^{\prime}, \\
b_{1}=-g^{\prime}, & f_{1}=-c^{\prime}, \\
c_{1}=-f^{\prime}, & g_{1}=-b^{\prime}, \\
d_{1}=e^{\prime}, & h_{1}=a^{\prime}
\end{array}
$$

whence $u_{1}=u^{\prime}$, that is $u^{\prime}=u^{3}$.

There is no difficulty in showing also, that if $a^{\prime \prime}, b^{\prime \prime}, \ldots h^{\prime \prime}$ are derived from $a^{\prime}, b^{\prime} \ldots h^{\prime}$, as these are from $a, b, \ldots h$, then

$$
a^{\prime \prime}=u^{2} a, \quad b^{\prime \prime}=u^{2} b, \ldots h^{\prime \prime}=u^{2} h .
$$

The particular case of this theorem, which corresponds to symmetrical values of the coefficients, is given by M. Eisenstein, Crelle, vol. XxvII. [1844], as a corollary to his researches on the cubic forms of numbers.

Considering this symmetrical case

$$
\begin{aligned}
& U=\alpha x^{3}+3 \beta x^{2} y+3 \gamma x y^{2}+\delta y^{3}, \\
& u=\alpha^{2} \delta^{2}-6 \alpha \delta \beta \gamma-3 \beta^{2} \gamma^{2}+4 \beta^{3} \delta+4 \alpha^{3} \gamma,
\end{aligned}
$$


so that if $U$ be transformed into

by means of

$$
U^{\prime}=\alpha^{\prime} x^{\prime 3}+3 \beta^{\prime} x^{\prime 2} y^{\prime}+3 \gamma^{\prime} x^{\prime} y^{\prime 2}+\delta^{\prime} y^{3},
$$

then if

$$
x=\lambda x^{\prime}+\mu y^{\prime} \text {, }
$$

we have

III.

$$
\begin{gathered}
y=\lambda, x^{\prime}+\mu, y^{\prime}, \\
u^{\prime}=\alpha^{\prime 2} \delta^{\prime 2}-6 \alpha^{\prime} \delta^{\prime} \beta^{\prime} \gamma^{\prime}-3 \beta^{\prime 2} \gamma^{\prime 2}+4 \beta^{\prime 3} \delta^{\prime}+4 \alpha^{\prime 3} \gamma^{\prime},
\end{gathered}
$$$$
u^{\prime}=(\lambda \mu,-\lambda, \mu)^{6} \cdot u \text {. }
$$

$$
p=3, \quad m=2, \quad n=4 \text {. }
$$

Notation as in I. $(C)$,

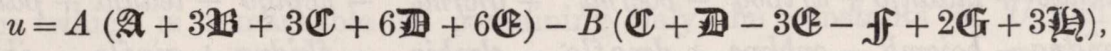

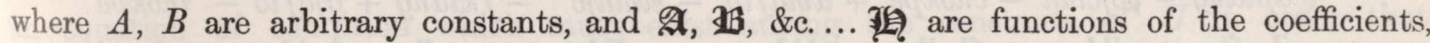
given as follows:-

$\mathcal{A}=a^{3} p^{3}-b^{3} o^{3}-c^{3} n^{3}+d^{3} m^{3}-e^{3} l^{3}+f^{3} k^{3}+g^{3} j^{3}-h^{3} i^{3}$.

$\mathbb{B}=-a^{2} p^{2} b o+b^{2} o^{2} a p+c^{2} n^{2} d m-d^{2} m^{2} c n+e^{2} l^{2} f k-f^{2} k^{2} e l-g^{2} j^{2} h i+h^{2} i^{2} g j$ $-a^{2} p^{2} c n+b^{2} o^{2} d m+c^{2} n^{2} a p-d^{2} m^{2} b o+e^{2} l^{2} g j-f^{2} k^{2} h i-g^{2} j^{2} e l+h^{2} i^{2} f k$

$-a^{2} p^{2} e l+b^{2} o^{2} f k+c^{2} n^{2} g j-d^{2} m^{2} h i+e^{2} l^{2} a p-f^{2} k^{2} b o-g^{2} j^{2} c n+h^{2} i^{2} d m$

$-a^{2} p^{2} h i+b^{2} o^{2} g j+c^{2} n^{2} f k-d^{2} m^{2} e l+e^{2} l^{2} d m-f^{2} k^{2} c n-g^{2} j^{2} b o+h^{2} i^{2} a p$.

$\mathfrak{C}=+a^{2} p^{2} d m-b^{2} o^{2} c n-c^{2} n^{2} b o+d^{2} m^{2} a p-e^{2} l^{2} h i+f^{2} k^{2} g j+g^{2} j^{2} f k-h^{2} i^{2} e l$

$+a^{2} p^{2} f k-b^{2} o^{2} e l-c^{2} n^{2} h i+d^{2} m^{2} g j-e^{2} l^{2} b o+f^{2} k^{2} a p+g^{2} j^{2} d m-h^{2} i^{2} c n$

$+a^{2} p^{2} g j-b^{2} o^{2} h i-c^{2} n^{2} e l+d^{2} m^{2} f k-e^{2} l^{2} c n+f^{2} k^{2} d m+g^{2} j^{2} a p-h^{2} i^{2} b o$.

四 = apbocn - apbodm-apcndm +bocndm-elfkgj +elfkhi +elgjhi - higjfk

+ apboel - apbojk - cndmgj $+d m c n h i-e l f k a p+e l f k b o+g j h i c n-h i g j d m$

- apbogj + apbohi + cndmel - dmcnkf + elfkcn - elfkdm-gjhiap + higjbo

+ apcnel - bodmfk - cnapgj + dmbohi-elgjap + fkhibo + gjelcn - hifkdm

- apcnfk + bodmel + cnaphi - dmboel + elgjbo - fkhiap - gjeldm + hifkcn

$-a p d m e l+b o c n k f+c m b o g j-d m a p h i+e l h i a p-f k g j b o-g j f k c n+h i d m e l$.

$\mathbb{E}=a p d m f k-b o c n e l-b o c n i h+d m a p g j-e l h i b o+f k a p g j+g j f k d m-h i e l c n$.

$\sqrt{\mathfrak{F}}=a^{2}$ phjo $-b^{2}$ goip $-c^{2} n f l m+d^{2} e m k n-e^{2} d l k n+f^{2} c k m l+g^{2} b j p i-h^{2} a i j o$

$-i^{2} p h b g+j^{2}$ ogah $+k^{2} n f d e-l^{2} m e c f+m^{2} l d c f-n^{2} c k d e-o^{2} b j a h+p^{2} a i b g$

$+a^{2} p h k n-b^{2}$ golm $-c^{2} n f i p+d^{2} e m j o-e^{2} d l j o+f^{2} c k i p+g^{2} b j p l-h^{2} a i n k$

$-i^{2} p h c f+j^{2} o g d e+k^{2} n f a h-l^{2} m e b g+m^{2} l d b g-n^{2} c k a h-o^{2} b j d e+p^{2} a i c f$

$+a^{2} p h l m-b^{2}$ golkn $-c^{2} n f j o+d^{2} e m i p-e^{2} l d p i+f^{2} c k j o+g^{2} b j n k-h^{2} a i m l$

$-i^{2} p h d e+j^{2} g o c f+k^{2} n f b g-l^{2} m e a h+m^{2} d l a h-n^{2} c k b g-o^{2} b j c f+p^{2} a i d e$

$+a^{2} p d n o-b^{2} c m p o-c^{2} b p m n+d^{2} a o m n-e^{2} h j k l+f^{2} g i l k+g^{2} f l i j-h^{2} e k j i$

$-i^{2} l f g h+j^{2} k e h g+k^{2} j h e f-l^{2} i g f e+m^{2} p b c d-h^{2} a o d c-o^{2} n d a b+p^{2} m b c a$

$+a^{2} p l n g-b^{2} h k m o-c^{2} e j p n+d^{2} f i o m-e^{2} c p j l+f^{2} d o i k+g^{2} a n l j-h^{2} b m k i$

$-i^{2} o d f h+j^{2} p c e g+k^{2} m h b f-l^{2} n a g e+m^{2} k h b d-n^{2} l g a c-o^{2} i f b d+p^{2} i e c a$

$+a^{2} p l f o-b^{2} e k p o-c^{2} h j m n+d^{2} g i m n-e^{2} b p k l+f^{2} a o l k+g^{2} d n i j-h^{2} c m j i$

$-\imath^{2} n d g h+j^{2} m c h g+k^{2} p b e f-l^{2} o a f e+m^{2} j h c d-n^{2} i g c d-o^{2} l f a b+p^{2} k e b a$. 


$$
\begin{aligned}
& \boldsymbol{G}=\text { apbgkn }- \text { boalhm }- \text { cndiep }+ \text { dmcjfo }- \text { elfocj }+ \text { flepied + gjhmla - ignble } \\
& \text {-ihjocf + gjidep + kflamh-lekbng + mdnbkg - ncmhal-obpied + paofjc } \\
& \text { + apbglm - boahkn - cndejo + dmcfip - elcfip + fkedoj + gjhakn-hibgml } \\
& \text {-ihjode + gijpcf + kflmbg - leknah + mdknah - nomlbg-obpicf + paojde } \\
& + \text { apcflg - bojehk - cnjehk + dmilgf - elmpbc + fkadno + gjadno-hipmcb } \\
& -i h a d n o+g j b m c p+k f c b p m-e l a d n o+\text { mdjehk - ncilfg - obilfg + pahejk } \\
& \text { + apcflm - bodkne - cnahjo + dmbgip - elbgip + fkahjo + gjednk - hicfml } \\
& -i h k n d e+m d a h j o+k f i p b g-b o c f m l+g j c f m l-n c p i g b-l e a h j o+p a k n d e \\
& \text { + apidng - bojcmh - cnbkpe + dmaflo - elmhjc - fkidng + gjaflo - ihbkpe } \\
& \text {-ihaflo + gjblkpe + fkcjhm - elidng + mdbkpe - cnaflo - boidng + pahmcj } \\
& \text { + apidfo - bojcep - nbkhm + cdmalng - elmnbk - fkalng + gjidfo - ihpecj } \\
& - \text { ihalng }+ \text { gjkbmh }+ \text { fkcjpe }- \text { elidfo }+ \text { mdjcep }- \text { ncidfo }- \text { obalng + pahmbk. } \\
& \text { F } \text { I }^{2}=a^{2} h o n l-b^{2} p g m k-c^{2} p f m j+d^{2} \text { onie }-e^{2} d p k j+f^{2} i l c o+g^{2} b l n i-h^{2} a m k j \\
& -i^{2} p d f g+j^{2} o e c h+k^{2} n b e h-l^{2} m a f g+m^{2} b l c h-n^{2} k a d g-o^{2} j a d f+p^{2} i b e c:
\end{aligned}
$$

we have, as usual,

$$
\dddot{u}=\left(\lambda_{1}{ }^{1} \lambda_{2}{ }^{2}-\lambda_{2}{ }^{1} \lambda_{1}{ }^{2}\right)^{3}\left(\mu_{1}{ }^{1} \mu_{2}{ }^{2}-\mu_{2}{ }^{1} \mu_{1}{ }^{3}\right)^{3}\left(\nu_{1}^{1} \nu_{2}{ }^{2}-\nu_{2}{ }^{1} \nu_{1}{ }^{2}\right)^{3}\left(\rho_{1}{ }^{1} \rho_{2}{ }^{2}-\rho_{1}{ }^{2} \rho_{2}\right)^{3} \cdot u .
$$

Particular forms of $U$ are

$$
\begin{aligned}
& A=1, \quad B=0 \text { : } \\
& u=\mathfrak{A}+3 \mathfrak{B}+3 \mathfrak{C}+6 \text { 珚 }+6 \mathfrak{C}=(a p-b o-c n+d m-e l+f k+g j-h i)^{3},=\nu \text { suppose. } \\
& A=1, B=9 \text { : } \\
& u=\mathfrak{A}+3 \mathfrak{B}-6 \mathfrak{C}-3 \text { 迅 }+33 \mathfrak{C}+9 \sqrt{\mathfrak{F}}-18 \mathfrak{G}-27 \mathfrak{T},=\theta U \text { suppose, }
\end{aligned}
$$

where $\theta U=0$ is the result of the elimination of the variables from the equations $d_{x_{1}} U=0, d_{y_{1}} U=0, d_{z_{1}} U=0, d_{w_{1}} U=0, d_{x_{2}} U=0, d_{y_{2}} U=0, d_{z_{3}} U=0, d_{w_{2}} U=0$. In fact, by an investigation similar to $\mathrm{Mr}$ Boole's, applied to a function such as $U$, it is shown that $\theta U$ has the characteristic property of the function $u$ : also in the present case $u$ is the most general function of its kind, so that $\theta U$ is obtained from $U$ by properly determining the constant. This has been effected by comparing the value of $u$, in the symmetrical case, with the value of $\theta U$, in the same case, the expanded expression of which is given by $\mathrm{Mr}$ Boole in the Journal, vol. IV. p. 169. Assuming $A=1$, the result was $B=9$. [Incorrect: the result of the elimination is not $\theta U=0$, but an equation of a higher degree.]

The general form of $u$ now becomes

$$
u=\alpha \nu^{3}+\beta \theta U,
$$

in which $\alpha, \beta$, are indeterminate.

$$
\begin{array}{lcc}
\text { We have } & \ldots u=\alpha \nu^{3}+\beta \theta \dddot{U}=M\left(\alpha \nu^{3}+\beta \theta U\right), \\
\text { where } & M=\left(\lambda_{1}{ }^{1} \lambda_{2}{ }^{2}-\lambda_{2}{ }^{1} \lambda_{1}{ }^{2}\right)^{3}\left(\mu_{1}{ }^{1} \mu_{2}{ }^{2}-\mu_{2}{ }^{1} \mu_{1}{ }^{2}\right)^{3}\left(\nu_{1}{ }^{1} \nu_{2}{ }^{2}-\nu_{2}{ }^{1} \nu_{1}{ }^{3}\right)^{3}\left(\rho_{1}{ }^{1} \rho_{2}{ }^{2}-\rho_{2}{ }^{1} \rho_{1}{ }^{2}\right)^{3} .
\end{array}
$$


and thence

$$
\nu^{3}=M \nu^{3},
$$

which coincides with a previous formula, and

$$
\theta \dddot{U}=M \theta U:
$$

whence, eliminating $\boldsymbol{M}$,

$$
\frac{\theta \dddot{U}}{\dddot{\nu}^{3}}=\frac{\theta U}{\nu^{3}}
$$

an equation which is remarkable as containing only the constants of $U$ and $\dddot{U}$ : it is an equation of condition which must exist among the constants of $\dddot{U}$ in order that this function may be derivable by linear substitutions from $U$.

In the symmetrical case, or where

$$
U=\alpha x^{4}+4 \beta x^{3} y+6 \gamma x^{2} y^{2}+4 \delta x y^{3}+\epsilon y^{4},
$$

it has been already seen that $\nu$ is given by

$$
\nu=\alpha \epsilon-4 \beta \delta+3 \gamma^{2} \text {. }
$$

Proceeding to form $\theta U$, we have

$$
\begin{aligned}
& \mathfrak{A}=\alpha^{3} \epsilon^{3}-4 \beta^{3} \delta^{3}+3 \gamma^{6} \text {, } \\
& \not B=4\left(\alpha \epsilon \beta^{2} \delta^{2}-\alpha^{2} \epsilon^{2} \beta \delta+3 \gamma^{2} \beta^{2} \delta^{2}-3 \beta \delta \gamma^{4}\right) \text {, } \\
& \mathfrak{C}=3\left(\alpha^{2} \epsilon^{2} \gamma^{2}+2 \gamma^{6}+\alpha \epsilon \gamma^{4}-4 \beta^{3} \delta^{3}\right) \text {, } \\
& \text { 烳 }=6\left(\alpha \epsilon \beta^{2} \delta^{2}-2 \alpha \epsilon \beta \delta \gamma^{2}+3 \beta^{2} \gamma^{2} \delta^{2}-2 \beta \delta \gamma^{4}\right) \text {, } \\
& \mathbb{E}=3 \alpha \in \gamma^{4}-4 \beta^{3} \delta^{3}+\gamma^{4} \text {, } \\
& \sqrt{f}=6\left(\alpha^{2} \delta^{2} \gamma \epsilon+\epsilon^{2} \beta^{2} \gamma \alpha-2 \beta^{3} \epsilon \gamma \delta-2 \delta^{3} \alpha \beta \gamma-4 \beta^{2} \gamma^{2} \delta^{2} .+4 . \beta \delta \gamma^{4}+\gamma^{3} \beta^{2} \epsilon+\gamma^{3} \alpha \delta^{2}\right) \text {, } \\
& \widetilde{G}=12\left(\alpha \epsilon \beta \delta \gamma^{2}-\alpha \beta \gamma \delta^{3}-\epsilon \gamma \delta \beta^{3}+\gamma^{3} \alpha \delta^{2}+\gamma^{3} \epsilon \beta^{2}+\beta \delta \gamma^{4}-2 \beta^{2} \gamma^{2} \delta^{2}\right) \text {, } \\
& \text { 政 }=\left(\alpha^{2} \delta^{4}+\epsilon^{2} \beta^{4}-4 \beta^{2} \epsilon \gamma^{3}-4 \alpha \gamma^{3} \delta^{2}+6 \beta^{2} \gamma^{2} \delta^{2}\right) \text {, }
\end{aligned}
$$

and these values give

$$
\begin{aligned}
& \theta U=\alpha^{3} \epsilon^{3}-6 \alpha \beta^{2} \delta^{2} \epsilon- 12 \alpha^{2} \beta \delta \epsilon^{2}-18 \alpha^{2} \gamma^{2} \epsilon^{2}-27 \alpha^{2} \delta^{4}-27 \beta^{4} \epsilon^{2}+36 \beta^{2} \gamma^{2} \delta^{2}+54 \alpha^{2} \gamma \delta^{2} \epsilon+54 \alpha \beta^{2} \gamma \epsilon^{2} \\
&-54 \alpha \gamma^{3} \delta^{2}-54 \beta^{2} \gamma^{3} \epsilon-64 \beta^{3} \delta^{3}+81 \alpha \gamma^{4} \epsilon+108 \alpha \beta \gamma \delta^{3}+108 \beta^{3} \gamma \delta \epsilon-180 \alpha \beta \gamma^{2} \delta \epsilon,
\end{aligned}
$$

so that this function, divided by $\left(\alpha \epsilon-4 \beta \delta+3 \gamma^{2}\right)^{3}$, is invariable for all functions of the fourth order which can be deduced one from the other by linear substitutions. The function $\alpha_{\epsilon}-4 \beta \delta+3 \gamma^{2}$ occurs in other investigations: I have met with it in a problem relating to a homogeneous function of two variables, of any order whatever, $\alpha, \beta, \gamma, \delta, \epsilon$ signifying the fourth differential coefficients of the function. But this is only remotely connected with the present subject.

Since writing the above, $\mathrm{Mr}$ Boole has pointed out to me that in the transformation of a function of the fourth order of the form $a x^{4}+4 b x^{3} y+6 c x^{2} y^{2}+4 d x y^{3}+e y^{4}$, besides his function $\theta u$, and my quadratic function $a e-4 b d+3 c^{2}$, - there exists a function 
of the third order $a c e-b^{2} e-a d^{2}-c^{3}+2 b d c$, possessing precisely the same characteristic property, and that, moreover, the function $\theta u$ may be reduced to the form

$$
\left(a e-4 b d+3 c^{2}\right)^{3}-27\left(a c e-a d^{2}-e b^{2}-c^{3}+2 b d c\right)^{2} ;
$$

the latter part of which was verified by trial; the former he has demonstrated in a manner which, though very elegant, does not appear to be the most direct which the theorem admits of. In fact, it may be obtained by a method just hinted at by $\mathrm{Mr}$ Boole, in his earliest paper on the subject, Mathematical Journal, vol. II. p. 70. The equations $d_{x}{ }^{2} u=0, d_{x} d_{y} u=0, d_{y}{ }^{2} u=0$, imply the corresponding equations for the transformed function: from these equations we might obtain two relations between the coefficients, which, in the case of a function of the fourth order, are of the orders 3 and 4 respectively: these imply the corresponding relations between the coefficients of the transformed function. Let $A=0, B=0, A^{\prime}=0, B^{\prime}=0$, represent these equations; then, since $A=0, B=0$, imply $A^{\prime}=0$, we must have $A^{\prime}=\Lambda A^{\prime}+\mathrm{M} B, \Lambda, M$, being functions of $\lambda, \lambda^{\prime}, \mu$, \&c. $\mu^{\prime}$ : but $B$ being of the fourth order, while $A, A^{\prime}$ are only of the third order in the coefficients of $u$, it is evident that the term $\mathrm{M} B$ must disappear, or that the equation is of the form $A^{\prime}=\Lambda A$. The function $A$ is obviously the function which, equated to zero, would be the result of the elimination of $x^{2}, x y, y^{2}$, considered as independent quantities from the equations $a x^{2}+2 b x y+c y^{2}=0, b x^{2}+2 c x y+d y^{2}=0$, $c x^{2}+2 d x y+e y^{2}=0$, viz. the function given above. Hence the two functions on which the linear transformation of functions of the fourth order ultimately depend are the very simple ones

$$
a e-4 b d+3 c^{2}, \quad a c e-a d^{2}-e b^{2}-c^{3}+2 b d c,
$$

the function of the sixth order being merely a derivative from these. The above method may easily be extended: thus for instance, in the transformation of functions of any even order, I am in possession of several of the transforming functions; that of the fourth order, for functions of the sixth order, I have actually expanded: but it does not appear to contain the complete theory. Again, in the particular case of homogeneous functions of two variables, the transforming functions may be expressed as symmetrical functions of the roots of the equation $u=0$, which gives rise to an entirely distinct theory. This, however, I have not as yet developed sufficiently for publication. There does not appear to be anything very directly analogous to the subject of this note, in my general theory: if this be so, it proves the absolute necessity of a distinct investigation for the present case, that which I have denominated the symmetrical one. 